\title{
Genome Sequence Resource of Phomopsis longicolla YC2-1, a Fungal Pathogen Causing Phomopsis Stem Blight in Soybean
}

\author{
Xiaolin Zhao, ${ }^{1,2,3}$ Zhichao Zhang, ${ }^{1,2,3}$ Sujiao Zheng, ${ }^{1,2,3}$ Wenwu Ye, ${ }^{1,2,3, \dagger}$ \\ Xiaobo Zheng, ${ }^{1,2,3}$ and Yuanchao Wang ${ }^{1,2,3}$ \\ ${ }^{1}$ Department of Plant Pathology, Nanjing Agricultural University, Nanjing, Jiangsu 210095, China \\ ${ }^{2}$ Key Laboratory of Plant Immunity, Nanjing Agricultural University, Nanjing, Jiangsu 210095, China \\ ${ }^{3}$ Key Laboratory of Integrated Management of Crop Diseases and Pests (Ministry of Education), \\ Nanjing, Jiangsu 210095, China
}

\begin{abstract}
Diaporthe-Phomopsis disease complex causes considerable yield losses in soybean production worldwide. As one of the major pathogens, Phomopsis longicolla T. W. Hobbs (syn. Diaporthe longicolla) is not only the primary agent of Phomopsis seed decay but is also one of the agents of Phomopsis pod and stem blight and Phomopsis stem canker. We performed both PacBio long-read sequencing and Illumina short-read sequencing and obtained a genome assembly for the strain $P$. longicolla YC2-1, which was isolated from soybean stem with Phomopsis stem blight disease. The 63.1 Mb genome assembly contains 87 scaffolds, with a minimum, maximum, and $N_{50}$ scaffold length of $20 \mathrm{~kb}$, 4.6 $\mathrm{Mb}$, and 1.5 Mb respectively, and a total of 17,407 protein-coding genes. The highquality data expand the genomic resource of $P$. longicolla species and will provide a solid foundation for a better understanding of their genetic diversity and pathogenic mechanisms.
\end{abstract}

\section{Genome Announcement}

Soybean is one of the best sources of plant-based protein and an important oilseed and cash crop worldwide. During soybean production, members of the fungal genus Diaporthe and its anamorph Phomopsis can cause a Diaporthe-Phomopsis disease complex including seed decay, pod and stem blight, and stem canker, leading to considerable yield losses both quantitatively and qualitatively (Baird et al. 2001; Li et al. 2010; Zorrilla et al. 1994). Among these fungi, the seed-borne pathogen Phomopsis longicolla T. W. Hobbs (syn. Diaporthe longicolla) is not only the primary agent of seed decay (Sinclair 1993) but is also one of the agents of pod and stem blight (Zhang et al. 1998) and stem canker (Mena et al. 2020). However, the mechanisms of $P$. longicolla development and pathogenicity in soybean are largely unknown.

The first genome assemblies of $P$. longicolla were published, in 2015, for strains TWH P74 and MSPL10-6, which were isolated from field-grown soybean seeds in Ohio in 1983 and in Mississippi in 2010, respectively. Based on Illumina sequencing, the genome assembly size of TWH P74 was $64.7 \mathrm{Mb}$, with 986 scaffolds and an $N_{50}$ scaffold length of $213.1 \mathrm{~kb}$ (Li et al. 2015a), and the genome assembly size of MSPL10-6 was $66.7 \mathrm{Mb}$, with 108

Xiaolin Zhao and Zhichao Zhang contributed equally to this work.

${ }^{\dagger}$ Corresponding author: W. Ye; yeww@njau.edu.cn

The author(s) declare no conflict of interest.

Accepted for publication 24 February 2021.

\section{Funding}

This work was supported by grants from the National Key R\&D Program of China (2018YFD0201000), the China Agriculture Research System (CARS-004-PS14), and the National Natural Science Foundation of China (31972250).

\section{Keywords}

Diaporthe, genome sequence, Phomopsis longicolla, soybean pathogen 
scaffolds and an $\mathrm{N}_{50}$ scaffold length of $1.04 \mathrm{Mb}$ (Li et al. 2015b). To expand the genomic resource and improve the quality of $P$. longicolla genomic data for a better understanding of the genetic diversity and pathogenic mechanisms, we sequenced the pathogenic strain $P$. longicolla YC2-1, which was isolated from a soybean stem with Phomopsis stem blight symptoms in Henan Province, China in 2019.

Genomic DNA of $P$. longicolla YC2-1 was extracted from a 2-day-old culture. Based on a library with fragment sizes of $>10 \mathrm{~kb}$, single molecule real time (SMRT) sequencing was performed on the Pacific Biosciences RSII sequencer (PacBio, Menlo Park, CA, U.S.A.), according to standard protocols (MagBead Standard Seq v2 loading, $1 \times 180 \mathrm{~min}$ movie), using the P4-C2 chemistry. After an error correction of raw reads using MECAT v2, a total of 8.2 Gb (approximately $130 \times$ coverage) of clean PacBio long reads were obtained, with an average length of $6.2 \mathrm{~kb}$, an $\mathrm{N}_{50}$ of $10.3 \mathrm{~kb}$, and read number of 1,322,662 (Table 1). A short-read sequencing for the extracted DNA was also performed using an Illumina NovaSeq 6000 system with insert sizes of approximately 300 to $400 \mathrm{bp}$ and a paired-end 150-bp strategy, and, finally, $6.1 \mathrm{~Gb}$ (approximately $92 \times$ coverage) of clean data with 40.4 million clean reads were generated. $k$-mer-based genome size estimation of the Illumina short reads, using GenomeScope v2.0 and KMC v3.1.1, revealed that the estimated genome size was $57.8 \mathrm{Mb}$, the proportion of repeat sequences was $6.2 \%$, and the heterozygosity was extremely low $(0.08 \%)$ (Table 1$)$.

The clean PacBio long reads attained from SMRT sequencing were used for de novo assembly using MECAT v2. The preassembled genome was further corrected for random errors in the long reads by aligning the Illumina short reads using Pilon v1.23. After a correction of 7,588 bp (substitution: $603 \mathrm{bp}$, deletion: 1,640 bp, and insertion: 5,345 bp) on the preassembled genome, the final genome assembly size of $P$. longicolla YC2-1 was $63.1 \mathrm{Mb}$, consisting of 87 scaffolds, with an $\mathrm{N}_{50}$ scaffold length of $1.5 \mathrm{Mb}$, a maximum length of 4.6 $\mathrm{Mb}$, a minimum length of $20.0 \mathrm{~kb}$, and an average length of $0.7 \mathrm{Mb}$ (Table 1). Compared with the two reported $P$. longicolla genome assemblies (TWH P74 and MSPL10-6), the assembly size was slightly reduced $(1.6$ to $3.6 \mathrm{Mb})$, the number of scaffolds was reduced by

Table 1. Genome features of Phomopsis longicolla YC2-1

\begin{tabular}{|c|c|}
\hline Features & Result \\
\hline \multicolumn{2}{|l|}{ PacBio long-read sequencing } \\
\hline Total length of clean reads & $8.2 \mathrm{~Gb}(130 \times)$ \\
\hline $\mathrm{N}_{50}$ length of clean reads & $10.3 \mathrm{~kb}$ \\
\hline Mean length of clean reads & $6.3 \mathrm{~kb}$ \\
\hline \multicolumn{2}{|l|}{ Illumina short-read sequencing } \\
\hline Total length of clean reads & $6.1 \mathrm{~Gb}(92 \times)$ \\
\hline k-mer estimated genome size & $57.8 \mathrm{Mb}$ \\
\hline$k$-mer estimated repetitive content & $6.2 \%$ \\
\hline$k$-mer estimated heterozygosity & $0.08 \%$ \\
\hline \multicolumn{2}{|l|}{ Genome assembly } \\
\hline Assembly size & $63.1 \mathrm{Mb}$ \\
\hline Scaffold number & 87 \\
\hline Scaffold $\mathrm{N}_{50}$ length & $1.5 \mathrm{Mb}$ \\
\hline Scaffold $L_{50}$ & 12 \\
\hline Mean scaffold length & $0.7 \mathrm{Mb}$ \\
\hline Maximum scaffold length & $4.6 \mathrm{Mb}$ \\
\hline Minimum scaffold length & $20.0 \mathrm{~kb}$ \\
\hline GC content & $48.7 \%$ \\
\hline BUSCO assessment (fungi_db10; $n=758$ ) & C:750, [S:746, D:4], F:3, M:5 \\
\hline \multicolumn{2}{|l|}{ Illumina reads mapping rate } \\
\hline Overall & $96.1 \%$ \\
\hline Properly paired & $93.2 \%$ \\
\hline \multicolumn{2}{|l|}{ Genome annotation } \\
\hline Proportion of repeat elements & $21.9 \%$ \\
\hline Protein-coding genes & 17,407 \\
\hline \multicolumn{2}{|l|}{ Ribosomal RNAs } \\
\hline 28s_rRNA & 5 \\
\hline 8s_rRNA & 35 \\
\hline 18s_rRNA & 5 \\
\hline Transfer RNAs & 247 \\
\hline
\end{tabular}


19.4 to $91.2 \%$, and the scaffold $\mathrm{N}_{50}$ length was increased to $1.4-$ to 7.0 -fold. Based on a reference dataset with 758 fungal benchmarking universal single-copy orthologs (BUSCOs), BUSCO v4.14 (Simão et al. 2015) identified 750 (98.9\%) complete BUSCOs, among which 746 were single-copy while only four were duplicated. The completeness of genome assembly was also evaluated by mapping Illumina short reads using HISAT2 v2.2.0, which showed that a total of $96.1 \%$ reads could be mapped and $93.2 \%$ reads were mapped and properly paired (Table 1).

Noncoding ribosome RNAs (rRNAs) prediction was carried out using RNAmmer (28s_rRNA $=5$; 8s_rRNA $=35 ; 18 s \_r R N A=5$ ) and transfer RNAs (tRNAs) were identified by tRNAscan-SE $(n=247)$. Repetitive elements identified by RepeatMasker v4.1.1 accounted for $21.9 \%$ of the genomic sequences (DNA transposons, $21.1 \%$; retrotransposons, $0.8 \%$ ). Repeat-masked genome assembly was applied for protein-coding gene prediction using GeneMark-ES v4.35, and 17,407 protein-coding genes were predicted (Table 1). Functional annotation of these genes was conducted using the National Center for Biotechnology Information nonredundant protein (16,279 genes, 93.5\%), Kyoto Encyclopedia of Genes and Genomes (14,496 genes, 83.3\%), Pfam (11,257 genes, 64.7\%), Gene Ontology (8,314 genes, 47.8\%), EuKaryotic Orthologous Groups (6,064 genes, 34.8\%), Pathogen Host Interactions (4,559 genes, 26.2\%), and Carbohydrate-Active EnZyme (2,766 genes, 15.9\%) databases. SignalP v4.0 predicted 2,089 proteins containing signal peptide $(12.0 \%)$, and antiSMASH v4.1.0 identified 1,315 genes (7.6\%) in 104 predicted secondary metabolic gene clusters (Table 1).

\section{Data Availability}

The genome sequencing raw data, assembly, and annotated genes reported in this paper have been deposited in the Genome Sequence Archive database (accession number subCRA004856) and the Genome Warehouse database (accession number GWHASJP00000000) in the National Genomics Data Center of China.

\section{Author-Recommended Internet Resources}

Kyoto Encyclopedia of Genes and Genomes database: https://www.genome.jp/kegg

SignalP: http://www.cbs.dtu.dk/services/SignalP

antiSMASH: https://antismash.secondarymetabolites.org

Carbohydrate-Active EnZYme database: http://www.cazy.org

Pathogen Host Interactions database: http://www.phi-base.org

\section{Literature Cited}

Baird, R. E., Abney, T. S., and Mullinix, B. G. 2001. Fungi associated with pods and seeds during the R6 and R8 stages of four soybean cultivars in southwestern Indiana. Phytoprotection 82:1-11.

Li, S., Darwish, O., Alkharouf, N., Matthews, B., Ji, P., Domier, L. L., Zhang, N., and Bluhm, B. H. 2015b. Draft genome sequence of Phomopsis longicolla isolate MSPL 10-6. Genom. Data 3:55-56.

Li, S., Hartman, G. L., and Boykin, D. L. 2010. Aggressiveness of Phomopsis longicolla and other Phomopsis spp. on soybean. Plant Dis. 94: 1035-1040.

Li, S., Song, Q., Ji, P., and Cregan, P. 2015a. Draft genome sequence of Phomopsis longicolla type strain TWH P74, a fungus causing Phomopsis seed decay in soybean. Genome Announc. 3:e00010-15.
Mena, E., Stewart, S., Montesano, M., and Ponce de León, I. 2020. Soybean stem canker caused by Diaporthe caulivora; pathogen diversity, colonization process, and plant defense activation. Front. Plant Sci. 10:1733.

Simão, F. A., Waterhouse, R. M., loannidis, P., Kriventseva, E. V., and Zdobnov, E. M. 2015. BUSCO: Assessing genome assembly and annotation completeness with single-copy orthologs. Bioinformatics 31:3210-3212.

Sinclair, J. B. 1993. Phomopsis seed decay of soybeans-A prototype for studying seed disease. Plant Dis. 77:329-334.

Zhang, A. W., Riccioni, L., Pedersen, W. L., Kollipara, K. P., and Hartman, G. L. 1998. Molecular identification and phylogenetic grouping of Diaporthe phaseolorum and Phomopsis longicolla isolates from soybean. Phytopathology 88:1306-1314.

Zorrilla, G., Knapp, A. D., and Mcgee, D. C. 1994. Severity of Phomopsis seed decay, seed quality evaluation, and field performance of soybean. Crop Sci. 34:172-177. 